\title{
Throughput analysis and real-time evaluation algorithm design of neighbor nodes for multi-hop mobile ad hoc network
}

\author{
Qingchao Zhu ${ }^{1,2, a, *}$ \\ ${ }^{1}$ School of Information Engineering, Engineering University of Chinese Armed Police Force, Xi'an, Shanxi 710086- China \\ ${ }^{2}$ School of Information and Navigation, Air Force Engineering University, Xi'an, Shanxi 710077-China \\ 'tgzy0516zqc@126.com
}

Abstract: Recently, distributed coordination function (DCF) protocol, standardized by IEEE 802.11 group, has played a dominant role in medium access control (MAC) layer of mobile ad hoc network (MANET). However, due to nodes mobility and topology dynamics, theoretical analysis models of DCF are limited to single-hop network. Unfortunately, mobility of neighbor nodes contended to access idle channel is unconsidered as well. Thus extension of throughput analysis model to multi-hop network and number evaluation algorithm design of neighbor nodes associated with mobility become two critical issues in MANETs. In this paper, we attempt to address the improvement of analysis model by integrating the Euclidean-Real-Ratio (ERR) and kalman filter algorithm into 2-dismension discrete time markov chain (DTMC). The contribution of this paper is threefold. First, we established a novel model to analyze throughput in multi-hop MANET based on definition of Euclidean-Real-Ratio (ERR). Second, we qualitatively show that $10 \%$ deviation occurs between theoretical analysis and simulation, reason of which is number variation of neighbor nodes. Third, we propose a real-time estimation methodology of neighbor nodes based on kalman filter algorithm coupled with redefinition of status update and measurement rules in poisson network(PN). Simulation results demonstrate the effectiveness and efficiency of our approach although it brings computation delay about $0.13 \mathrm{~s}$.

Keywords: mobile ad hoc network; throughput; medium access control; poisson distribution; kalman filter algorithm

\section{Introduction}

Mobile ad hoc network (MANET) is an autonomous wireless network system without fixed infrastructure but independent nodes, which play a role as both terminal and router [1,2]. Since protocol of MAC layer could not only interact with physical channel directly, but also provide interface to upper layer, design, analysis and optimization of it have been focused for decades and distributed coordination function (DCF) protocol [3,4] becomes standard of MAC layer recommended by IEEE802.11 group. However, absence of infrastructure introduces new challenges, such as theoretical analysis model [5], security risks [6], fairness [7], energy consumption [8] and cognitive MAC [9]. These challenges impede the large-scale application of MANET and depend on optimization of theoretical model, which has limited to single-hop network and many assumptions, not suitable for MANET. Moreover, the triplet of throughput, delay and reliability (TDR) [10] forms a comprehensive metric of performance and the focus of this paper is throughput.

Several techniques such as 2-D discrete time markov chain[11], poisson point process[12], mobility model[13] can be implemented on theoretical throughput analysis. However, they are not guaranteed to be successful, complexity may make analysis more and more difficult, and influence of mobility in MANET isn't considered. An appropriate solution to these issues will be integrating statistical analysis method into multi-hop model, which is a primary challenge because of node mobility and stationary distribution. The former two factors will alter contention nodes number $N$ and access probability $p$ potentially but both are always assigned to constant values, which diminish accuracy of recent models. For example, focusing on the case where nodes are distributed on the 2-D plane as a homogeneous poisson network (PN) and $N$ is initialized to $N_{0}$ and after $t$ seconds $N$ would be a random variable $N_{t}$ because of node mobility. 
Equation $N_{0}=N_{t}$ couldn't be held all the time. Therefore $N$ should be changed over time as well as $p$. Unfortunately, none of existing theoretical models have taken it into consideration at all, making their applicability in MANET rather doubtful.

In this paper, focusing on throughput model improvement, we tackle the above issues in three steps. First, we extend the 2-D DTMC model into multi-hop MANET without consideration of mobility through definition of Euclidean- Real-Ratio (ERR), based on poisson distribution, saturated traffic, ideal channel, constant contention nodes. After this, we reveal the fact qualitatively that difference between theory and simulation is resulted from nodes mobility through following the tracks of trace and nam files, which may alter parameters like $N$ and $p$ while no work has analyzed about it. Second, we take mobility into consideration and propose a neighbor estimation algorithm to enhance accuracy based on kalman filter through redefinition of state update and measurement rules. Third, we show the results by comparing extended model to simulation. Results show that extended model considered mobility outperforms DTMC by $8 \%$ in terms of accuracy.

The remainder of this paper is outlined as follows. Section 2 summarized the related work. In Section 3, an improved model of DTMC is proposed for multi-hop MANETs. Section 4 describes the number estimation methodology of neighbor nodes based on kalman filter algorithm considering mobility. We present numerical results of the new model in Section 5. Section 6 concludes the paper.

\section{Related Work}

The most popular method to analyze DCF protocol is 2-D DTMC based on two stochastic processes $b(t)$ and $s(t)$, representing backoff time counter and backoff stage respectively. It is also called Bianchi model[11] for both two-way handshaking mechanism and four-way handshaking technique known as virtual carrier sense(VCS)[3], on basis of certain assumptions like saturated traffic, ideal channel, unlimited retransmission and Boolean logic for collision [14,15]. Deviation between theory and simulation is less than 0.1 percent, and its higher accuracy makes it be basic of new models, the overwhelming majority of which focus on improvement of Bianchi model in certain aspects. However, algorithms based on DTMC have two similar limitations. One is single-hop nature, which is unsuitable for multi-hop MANET. The other is static nature without consideration of dead or new nodes. Both shortages restrict the application of DTMC. The key issue is statistical analysis of nodes distribution.

In network distribution, Baroudi et al [16] investigate the impact of sensor node distributions to performance of different flavors of LEACH algorithm to optimize the assumption of uniformly distributed sensor nodes, and result proves that poisson distribution of network nodes perform better than uniform distribution. LIU et al. [17] present a detailed analysis to random direction model in square region, show that node distribution probability is higher and higher as its location varies by the sequence of center, border, and corner, and the stationary state of nodes approaches to exponential distribution. Nombuso et al. [18] present that nodes in application scenarios of MANET could be distributed either in a uniform or normal fashion, and they analyze AODV, OLSR and HWMP protocols in normal and uniform distributions, whose results demonstrate that poisson distribution of nodes is more practical and feasible. Marimon et al. [19] verify the correctness of poisson distribution in oceanography quantitatively, which provides a reference for analysis of network distribution. Therefore, there is no doubt that analyzing throughput performance of Poisson Network (PN) is significant and new model will focus on it.

Besides, to analyze throughput of multi-hop network, Chiang et al. [20] describe the implementation of software synchronization mechanism operated on multi-hop MANET without any hardware/protocol modifications, demonstrate its effectiveness in DCF, and establish foundation in theoretical analysis of multi-hop network. Navid et al. [15] explain that throughput analysis of multi-hop MANET is challenging and it hasn't been accomplished in the literature. An alternative probabilistic modeling approach is developed, which ignores mobility. Muhammad et al. [21] propose a spatial poisson point process model for multi-hop 
MANET with fixed boundaries, which relies on probability density function of received power, and network coverage is calculated for different set of parameters. Though aforementioned works haven't considered influence of mobility, they are crucial for analyzing throughput of multi-hop MANET.

In summary, DTMC model isn't suitable for MANET. Thus we develop a novel model for VCS called RTS/CTS/DATA/ACK mechanism via definition of Euclidean-Real-Ratio (ERR) for MANET, whose nodes are deploy in a PN as discussed above. Moreover, it's time we introduced number estimation algorithm aiming at mobility into theoretical model by kalman filter, which would enhance accuracy of the model.

\section{Proposed Multi-hop Analysis Model}

In this section, we provide a novel model based on Euclidean- Real-Ratio (ERR) for VCS to extend 2-D DTMC to multi-hop MANET with nodes deployed in PN.

\subsection{Multi-hop analytical model}

According to $[3,11]$, to analyze throughput of VCS based on RTS/CTS/DATA/ACK, several additional assumptions needed for DTMC and new model are as follows.

1) Boolean logic for collision occurrence, which presumes that transmissions either collide or are successful;

2) Saturated traffic condition, that is, packet will generate after a successful completion of a DATA packet;

3) Unlimited number of retransmissions, which guarantee that all packets are received ultimately, and that each packet collides with constant and independent probability $p$;

4) Ideal channel, which ignores interference and noise.

2-DTMC model for single-hop network proposed by Bianchi [11] is formulated as

$$
S=\frac{p_{s} p_{t} E[P]}{\left(1-p_{t r}\right) \sigma+p_{s} p_{t r} T_{s}+p_{t r}\left(1-p_{s}\right) T_{c}}=\frac{p_{s} p_{t r} E[P]}{E[\sigma]}
$$

where $N$ denotes number of nodes contending for idle channel, $H$ equals total bits of PHY and MAC header, $\delta$ denotes propagation delay, RTS , CTS , SIFS, DIFS , ACK denote transmission delay of corresponding packets in VCS. Specifically, all of them are assigned constant values. Besides, other symbols like $p, p_{t r}, \tau, p_{s}, t_{s}, t_{c}$ denote access probability, transmission probability, access delay, success transmission probability, success transmission period and collision time. It's notable that they are variables, partial of which are computed as follows.

$$
\begin{aligned}
& p_{s}=N \tau(1-\tau)^{N-1} / 1-(1-\tau)^{N}, p=1-(1-\tau)^{N-1}, p_{t r}=1-(1-\tau)^{N}, T_{c}=R T S+D I F S+\delta, \\
& \left.\tau=\frac{2(1-2 p)}{(1-2 p)(W+1)+p W\left(1-(2 p)^{m}\right)}\right)^{\text {function }}=\frac{2}{=} \frac{2}{W+1+p W \sum_{i=0}^{m-1}(2 p)^{i}}, T_{s}=R T S+S I F S+\delta+C T S+S I F S+\delta+H+E[P]+S I F S+\delta+A C K+D I F S+\delta
\end{aligned}
$$

The eq. (1) is the popular 2-D DTMC model. However, it is limited to single-hop network, lacking consideration of multi-hop nature and mobility. How to extend it into MANET is an issue.

To realize it, Euclidean distance between source and destination and real total distance should be considered, former of which is a random variable, never been brought into Bianchi model or its improved ones, resulting in unsuitable for multi-hop network. On contrast, the latter may be obtained from route table. Since Euclidean distance is shorter than real distance, establishing the relationship of both parameters is essential. Therefore, we define a random variable called Euclidean- Real-Ratio (ERR) as the ratio of Euclidean distance and total distance, and the larger ERR is, the better new model performs, which imply that approximation of both will be higher, but it could never be larger than unity. Fig.1 illustrates a diagram of $n$ hops link between source and destination, in which $d_{i}$ denotes Euclidean distance of $i$ th hop and $\varphi_{i}$ represents angle between horizontal line and $i$ th link. Nakagami- $m$ distribution of $d_{i}$ in PN has been demonstrated [22,23]. Therefore ERR (denoted by $\gamma$ ) is formulated as

$$
\gamma=\frac{d}{\sum_{i=1}^{n} d_{i}}=\frac{\sqrt{\left(\sum_{i=1}^{n} d_{i} \cos \varphi_{i}\right)^{2}+\left(\sum_{i=1}^{n} d_{i} \sin \varphi_{i}\right)^{2}}}{\sum_{i=1}^{n} d_{i}}=\frac{\sqrt{\sum_{i=1}^{n} d_{i}^{2}+2 \sum_{i=1}^{n-1} \sum_{j=1+1}^{n} d_{i} d_{j} \cos \left(\varphi_{i}-\varphi_{j}\right)}}{\sum_{i=1}^{n} d_{i}}
$$


A simplified computing formulation by making a square of Eq. (2) is defined as

$$
\gamma^{2}=\frac{d^{2}}{\left(\sum_{i=1}^{n} d_{i}\right)^{2}}=\frac{\sum_{i=1}^{n} d_{i}^{2}+2 \sum_{i=1}^{n-1} \sum_{j=i+1}^{n} d_{i} d_{j} \cos \left(\varphi_{i}-\varphi_{j}\right)}{\left(\sum_{i=1}^{n} d_{i}\right)^{2}}
$$

Since $d_{i}$ and $\varphi_{i}$ are random variables, $\gamma^{2}$ is a random variable obviously. So choosing $E\left[\gamma^{2}\right]$ will be better than $\gamma^{2}$, because a larger $\gamma^{2}$ variations will lead to an unbearable throughput deviation. Therefore

$$
E_{d_{i, \varphi_{i}}}\left(r^{2}\right)=E_{d_{i}}\left(E_{\varphi_{i}}\left(r^{2} \mid d_{i}\right)\right), i=0,1,2, \cdots, n
$$

Based on assumption of PN [24] and law of Large Numbers, $\varphi_{i}$, where $i=0,1,2, \cdots, n$, can be approximated with its probability density function (PDF) as

$$
\varphi_{i} \sim\left(0, \frac{(\pi+\theta)^{2}(i-1)+\theta^{2}}{12}\right)
$$

where $\theta_{i}$ denotes the angle between sender destination line and sender to a next-hop node, following a uniform distribution and being confined to $[-\theta / 2, \theta / 2]$. For $\varphi_{i}$, we also have

$$
E_{\varphi_{i}, \varphi_{j}}\left(\cos \left(\varphi_{i}-\varphi_{j}\right) \mid d_{i}\right)=e^{-(\pi+\theta)^{2}(i-1)+\theta^{2} / 12}
$$

Plugging (5) and (6) into (4), we obtain an improved expression for $E\left[\gamma^{2}\right]$ defined as

$$
E_{d_{i}, \varphi_{i}}\left(r^{2}\right)=\underbrace{E_{d_{i}} \frac{\sum_{i=1}^{n} d_{i}^{2}}{\left(\sum_{i=1}^{n} d_{i}\right)^{2}}}_{I_{1}}+2 \underbrace{e_{\sigma_{i}^{2}}^{2} E_{d_{i}} \sum_{i=1}^{n-1} \sum_{j=i+1}^{n} \sum_{i=1}^{n} d_{i} d_{j}}_{I_{2}}
$$

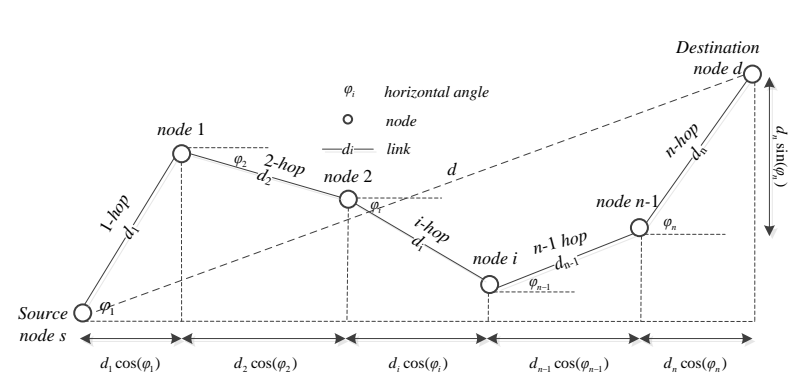

Fig. 1. Schematic diagram of $n$ hops in a PN between source and destination where calculation procedure is split into two parts.Since $d_{i}$ is Nakagami-m distributed in a PN [25], $v_{i}=d_{i}^{2}$ obeys a gamma distribution. With this characterization, variable $I$ is simplified as

$$
I_{1}=E_{d_{i}} \frac{\sum_{i=1}^{n} d_{i}^{2}}{\left(\sum_{i=1}^{n} d_{i}\right)^{2}}=\sum_{i=1}^{n} E_{d_{i}}\left(\frac{V_{i}}{J}\right)=n E_{d_{i}}\left(\frac{v_{i}}{J}\right)
$$

Using Jacobian algorithm and assuming $v_{i}$ and $J$ to be independent random variables, both of which obey gamma distribution, we get a close-form expression of Eq. (8), as shown in the following

$$
\left.I_{1}=n E_{d_{i}}\left(\frac{g_{i}}{W}\right)\right)^{n>2} \approx \frac{n m^{2}}{n m-1} \cdot \frac{1}{m+(n-1)\left(\frac{\Gamma(m+1 / 2)}{\Gamma(m)}\right)^{2}}
$$

Similarly, second term $I_{2}$ in (7) can be calculated by

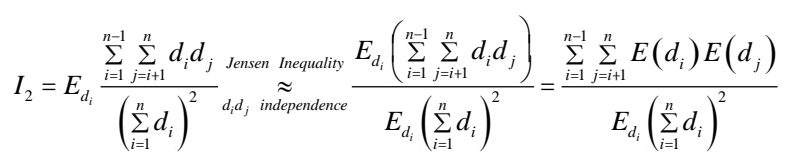

By substituting (9) and (10) in (7), we can obtain the expression of $E\left[\gamma^{2}\right]$.

Then we construct the relationship of channel idleness probability $p_{\text {idle }}$, successful transmission probability $p_{s}^{\prime}$ and transmission probability $\tau$ with $E\left[\gamma^{2}\right]$. For PN, channel is sensed as idle if two conditions are 
satisfied. The one is that, none of neighbor nodes in the sensing range of sender transmits RTS packet, represented as $\mathrm{C} 1$. The other one is that, none of neighbor nodes in the intersection of hearing ranges of receiver sends CTS packet in response to RTS, which is denoted as C2.Thus $p_{\text {idle }}$ is defined as

$$
p_{\text {idle }}=P\left(C_{1} \cap C_{2}\right)=P\left(C_{1}\right) \cap P\left(C_{2}\right)
$$

According to $[24,26]$, probability of no nodes transmits RTS in specified slot is equal to

$$
P\left(C_{1}\right)=e^{-i \pi R^{2} t}
$$

where $\lambda$ denotes intensity of PN, $R$ represents transmission range. Similarly, $P\left(C_{1}\right)$ is formulated as

$$
P\left(C_{2}\right)=e^{-1.3 .3 R^{2} \cdot \exp \left(-1.3 .3 R^{2}\right)}
$$

Therefore plugging (12) and (13) into (11), $p_{\text {idle }}$ can be rewritten as

$$
p_{\text {idle }}=\exp \left\{-\lambda \tau R^{2}\left(\pi+1.3 \exp \left[-1.3 \lambda \tau R^{2}\right]\right)\right\}
$$

From [11], $\tau$ and $p_{\text {idle }}$ are dependent on each other according to $\tau=2\left(1-2\left(1-p_{\text {idde }}\right)\right) / W_{\text {init }}$, where $W_{\text {init }}$ stands for initial length of backoff window. Thus $\tau$ could be achieved as follows.

$$
\tau=\frac{2}{W_{\text {init }}}\left\{1-2\left\{1-\exp \left\{-\lambda \tau R^{2}\left(\pi+1.3 \exp \left[-1.3 \lambda \tau R^{2}\right]\right)\right\}\right\}\right\}
$$

As stated previously, $R$ obeys a Nakagami- $m$ distribution, where optimal value of $m$ is calculated by maximizing $E\left[\gamma^{2}\right]$, which is formulated as

$$
m^{\prime}=\max E\left[\gamma^{2}\right]
$$

Plugging (15) and (16) into (1), we obtain

$$
\left\{\begin{array}{l}
P_{t r}^{\prime}=\min p_{t r, i}, p_{t r}=1-(1-\tau)^{N} \\
P_{s}^{\prime}=\min p_{s, i}, p_{s, i}=\frac{N \tau(1-\tau)^{N-1}}{1-(1-\tau)^{N}}
\end{array}\right.
$$

where $N$ denotes number of neighbor nodes. Thus throughput of multi-hop network is established by (1), (15) and (17).

\subsection{Qualitative deviation analysis of neighbor nodes}

In this subsection, we first compare analytical throughput of ERR model with simulation by NS2 [27]. Then we reveal influence of mobility in forms of neighbor nodes qualitatively, which points out the necessity of introducing number nodes estimation algorithm in Section 4.

\subsubsection{Network settings}

Here, we adopt similar model as previous work with unique difference of walk nodes, in which velocity is assigned to $2 \mathrm{~m} / \mathrm{s}$ in PN with range of $5 \mathrm{~km} \times 5 \mathrm{~km}$. The mobility model of Random Walk is used. DSR (Dynamic Source Routing) protocol and CBR (Constant Bit Rate) traffic of 1450Bytes, with rates as 11Mbps, are set to network and application layer, respectively. Max number of connection is set to 10. Contention window is initialized to 32. Other parameters standardized by IEEE 802.11 group are as shown in table 1 . We run our simulations for 600s with three instances, so that node distribution can reach a stable state.

Table 1. Standard settings of DCF by IEEE 802.11 group

\begin{tabular}{cc|cc}
\hline parameter & value & parameter & value \\
\hline ACK expired time & $300 \mu \mathrm{s}$ & Propagation delay & $1 \mu \mathrm{s}$ \\
CTS bits & $112 \mathrm{bit}+\mathrm{PHY}$ & slot length & $50 \mu \mathrm{s}$ \\
CTS expired time & $300 \mu \mathrm{s}$ & DIFS & $128 \mu \mathrm{s}$ \\
RTS bits & $160 \mathrm{bit}+\mathrm{PHY}$ & SIFS & $28 \mu \mathrm{s}$ \\
Header bits & MAC:272bit & ACK & $112 \mathrm{bit}+\mathrm{PHY}$ \\
& PHY:128bit & & \\
\hline
\end{tabular}

Fig. 2 illustrates the results and we summarize key observations. From the figure, as number of nodes increase, throughput of both methods increase owing to that, more and more couples of nodes participate in 
communication, while theoretical analysis achieve larger values than simulation, since the former method ignores certain factors, which are discussed in next subsection. Besides, throughput of both methods stays at a stable value with increasing because total couples of transmit-receive nodes remain almost unchanged. Therefore, efficiency along with feasibility of ERR is validated here. However, theoretical throughput is about 4.4Mbps, 10 percent higher than simulation. Reasons like fairness, noise, channel capacity, dynamic neighbor nodes have been focused. The first three issues have been researched. But to the best of our knowledge, the last one hasn't been accomplished in the literature. To fulfill it, we explain it qualitatively and propose a new methodology in next Section.

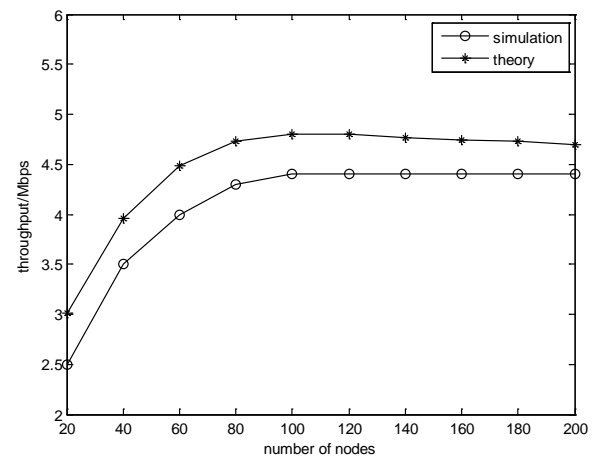

Fig. 2. Throughput comparison between simulation and theoretical analysis

\subsubsection{Qualitative analysis of deviation}

Aiming at interpretation, events in trace file of NS2 should be analyzed, so we abstract two periods, 14.59s 14.95s and 28.5s 28.75s, and statistical results are shown in Fig.3. In theory, after a successful contention for idle channel, once RTS/DATA are transmitted to the destination, CTS/ACK should be received by source node. However, Fig.3 illustrates that, a few control packets still exist after a successful transmission of DATA and a large disparity of RTS number arises in 14s and 28s. Two reasons blaming for them could be concluded, one of which source has never received CTS from the destination, which dropped RTS packets because of expiration, and the other of which new joined nodes may not detect any packets from neighbor nodes before sending RTS to contend for channel. To confirm it, we observe the statistical results of RTS packets and nodes distribution at 14s and 28s through trace file and nam tools, shown in Fig. 4 and Fig. 5 respectively. Retransmission occurs only if there are more than two RTS packets of the same node in adjacent slots. Here we pay attention to node 5, 8, 23, 24 and 26 in the same area of PN.

From Fig. 4, total packets of RTS delivered by node 5 and 8 are 2 and 3 respectively among the period of 14.59s to 14.95s, denoted by symbol R in trace file. Therefore, identical RTS packets don't result from expiration, make the first reason false. Moreover, according to CSMA/CA mechanism, source sends RTS or NAV packets to tell its neighbors to enter the backoff period after contending idle channel successfully. However, the facts are just opposite because new joined nodes sensed channel to be idle for unhearing RTS will transmit RTS packets, resulting in collision and retransmission in next slot in accord with the second reason. Fig. 5 demonstrates this conclusion. Three nodes $(5,8,26)$ are neighbors of node 24 at $14 \mathrm{~s}$ in Fig.5(a), but due to mobility, only node 8 locates around it in Fig.5(b). Therefore, RTS numbers of the latter are less than the former obviously, which matches to Fig.3 and Fig.4. Therefore, necessity of introducing number estimation algorithm of neighbor nodes is undoubted. 


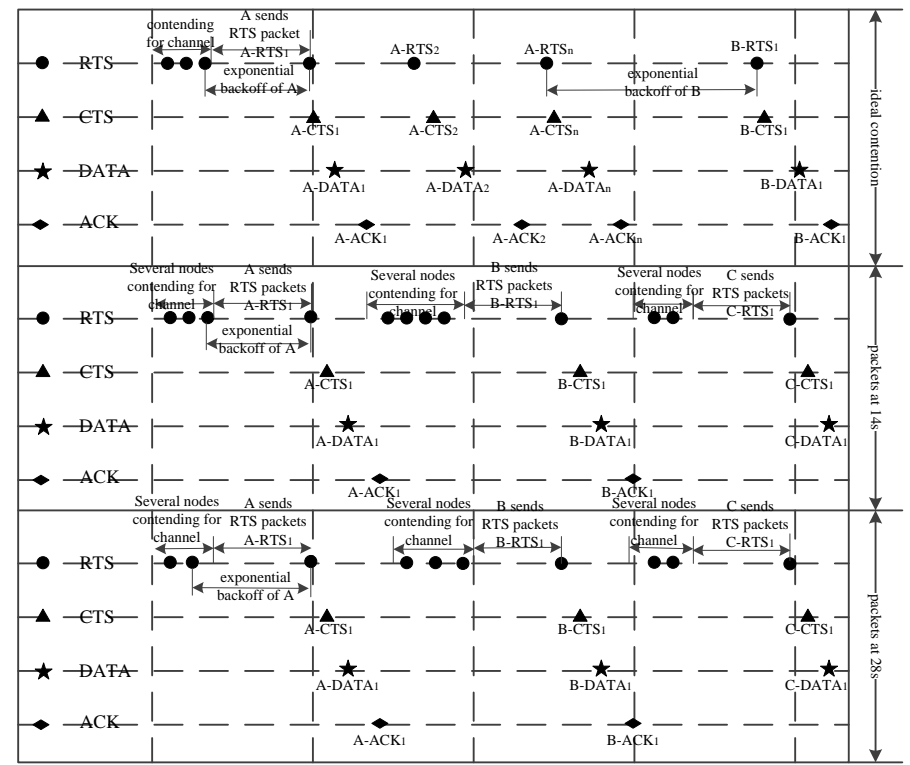

Fig. 3 Statistics of corresponding packets at different moments

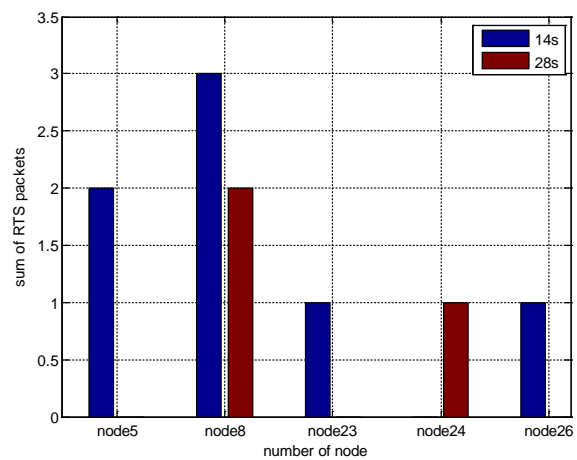

Fig. 4 Sum of RTS packets at 14s and 28s of different nodes

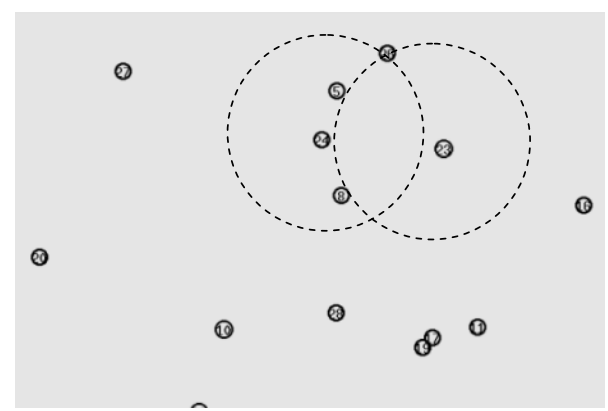

(a)Nodes distribution at $14 \mathrm{~s}$

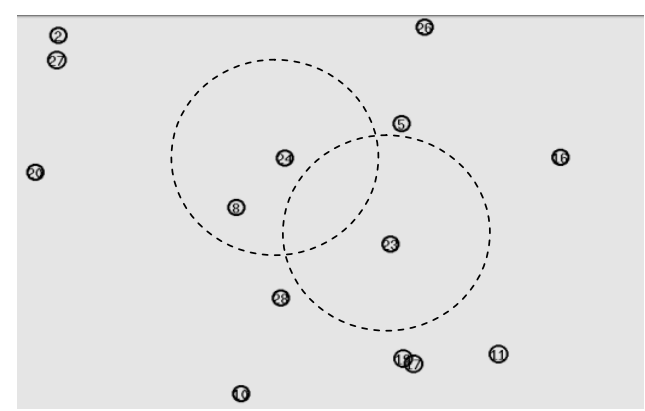

(b) Nodes distribution at $28 \mathrm{~s}$

Fig. 5 Nodes distribution at different moments

\section{Design of Real-time Estimation Algorithm of Neighbor Nodes}

In this section, we will propose a real-time estimation algorithm based on kalman filter, with redefinitions of state update rule and measurement rule to match with multi-hop MANET, whose nodes are distributed in a PN.

To realize it, from Eq. (1), inverse function of $N$ with other parameters of DCF can be expressed as

$$
N=f(p)=1+\frac{\log (1-p)}{\log \left(1-\frac{2(1-2 p)}{(1-2 p)(W+1)+p W\left(1-(2 p)^{m}\right)}\right)}
$$


where $m$ denotes maximum backoff stage in 2-D DTMC, and $w$ represents minimum contention window. As we know, $m$ and $w$ are constant parameters. In fact, it provides an explicit equation of $N$ versus $p$, $m, w$. Specially, $p$ could be estimated by any node independently based on the assumptions through monitoring sharing channel activity. Hence, estimation of $p$ is equivalent to $N$ in Eq. (18). Since $p$ is defined as the failure probability of transmitted packets in certain slot, that is, more than two parallel transmissions occur, which could be divided into two segments, called as number of experienced collisions $N_{\text {coll }}$ and number of busy slots $N_{\text {busy }}$. Let $N_{\text {toall }}$ denote total number of slots. Thus $p$ is calculated as

$$
p=\frac{N_{\text {coll }}+N_{\text {busy }}}{N_{\text {total }}}
$$

where $N_{\text {coll }}, N_{\text {busy }}$ and $N_{\text {toal }}$ are highly dependent on history records, which should be improved to meet the need of real-time estimation. Fortunately, kalman filter[28] could solve the issue for its efficiency, so that we integrate it into the new algorithm.

We discretize time in $N_{\text {toal }}$ parts (a predefined constant value) with length of $E[\sigma]$ (statistical variable). In the $k$ th slot, let $p_{k}$ denote the relative conditional collision probability. It is defined as

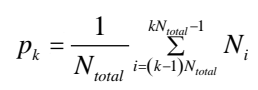

where equation $N_{i}=0$ is satisfied only if $k$ th slot is empty or transmission is success, with probability of $P\left(N_{i}=0\right)=1-p$. Otherwise, $N_{i}=1, P\left(N_{i}=1\right)=p$. Therefore, random variable $p_{k}$ obeys binomial distribution, which is written as follows.

$$
P\left(p_{k}=\frac{i}{N_{\text {total }}}\right)=\left(\begin{array}{c}
N_{\text {total }} \\
i
\end{array}\right) p^{i}(1-p)^{N_{\text {soul }}-i}
$$

Based on Eq. (21), it's obvious that mean value and variance of $p_{k}$ are calculated as $E\left[p_{k}\right]=p$ and $\sigma\left[p_{k}\right]=p(1-p) / N_{\text {total }}$, respectively.

As mentioned earlier, it's necessary to redefine two rules to estimate the number of neighbor nodes, called as system states update rule and measurement rule. In our algorithm, the system states are trivially denoted by number symbol $N_{k}$ of nodes at discrete time $k$ as shown in Eq. (20). Therefore, node performs real-time update rule at any moment as

$$
N_{k}=N_{k-1}+\Omega_{k}
$$

where number $N_{k}$ of neighbor nodes at time $k$ is calculated by that at time $k-1$ plus a random variable $\Omega_{k}$ called state noise.

Regarding to measurement rule, since $p_{k}$ can be expressed by the inverse function of $N_{k}$ through Eq. (5), which is denoted as $h\left(N_{k}\right), p_{k}$ in Eq. (20) is rewritten as

$$
p_{k}=f^{-1}\left(N_{k}\right)+v_{k}=h\left(N_{k}\right)+v_{k}
$$

where $v_{k}$ is a random variable which obeys binomial distribution with zero mean and variance of $h\left(N_{k}\right)\left[1-h\left(N_{k}\right)\right] / N_{\text {total }}$ as Eq. (21).

Equations (22) together with (23) provide a complete description of state model, based on which we propose a novel algorithm to estimate number of neighbor nodes at any time.

Let $\hat{N}_{k}$ and $\sigma_{k-1}$ represent estimated value of $N_{k}$ and deviation between the two variables. According to kalman filter algorithm and Eq. (21), we obtain

$$
\hat{N}_{k}=\hat{N}_{k-1}+K_{k} z_{k}
$$

where $z_{k}$ denotes the innovation value by $k$ th measure, calculated as $z_{k}=p_{k}-h\left(\hat{N}_{k-1}\right)$, where $K_{k}$ represents Kalman gain, which is expressed as 


$$
K_{k}=\frac{\left(\sigma_{k-1}+Q_{k}\right) h_{k}}{\left(\sigma_{k-1}+Q_{k}\right) h_{k}^{2}+R_{k}}
$$

where $Q_{k}$ denotes the variance of $\Omega_{k}$ and is assigned to constant values like $0,0.1$ and 0.001 for different systems or networks, thus $Q_{k}$ is replaced by $Q$ for simplicity; $R_{k}$ represents the variance of $p_{k}$, which is equal to $R_{k}=h\left(\hat{N}_{k-1}\right)\left(1-h\left(\hat{N}_{k-1}\right)\right) / N_{\text {total }} ; \quad h_{k}$ denotes sensitivity of measurement rule, computed by $\partial h(n) /\left.\partial n\right|_{n=\hat{n}_{k-1}}$; $\sigma_{k}$ represents error variance expressed as $\sigma_{k}=\left(1-K_{k} h_{k}\right)\left(\sigma_{k-1}+Q_{k}\right)$; random variable $\Omega_{k}$ equals to $1 / R_{k}$. Plugging these values and (25) together with (22) and (23) into (24), we fulfill the real-time number estimation of neighbor nodes denoted by $\hat{N}_{k}$. By substituting $\hat{N}_{k}$, (16) and (17) into Eq. (1), we obtain the improved throughput analysis model of ERR for multi-hop MANET considered mobility through $\hat{N}_{k}$.

\section{Numerical Results}

In this section, we present the deviation between ERR with estimation algorithm and simulation. Results will demonstrate correctness and efficiency of the novel algorithm.

All the parameters are completely same with that in subsection 3.3.1 except introducing number estimation of neighbor nodes algorithm here. Each simulation is performed three instances and we take the average values as the final results shown in Fig. 6. Moreover, we also list the disadvantage of computation delay in Table.2 with different sizes of packets, which is introduced by estimation algorithm for further research.

From the figure, we observe that theoretical results are similar to simulation, which is better than Fig. 2 and accuracy of our proposed theoretical model is 8 percent higher than ERR without consideration of mobility through neighbor nodes. Thus this outcome not only proves correctness of analysis on the first reason, but also demonstrates the efficiency of the novel model based on ERR with mobility. However, it introduces a little computation delay about 0.13 s as listed in Table. 2 , which is brought by estimation algorithm and could be optimized through extended kalman filter, which is not discussed in this article, so that the pros and cons should be weighed when it will be used.

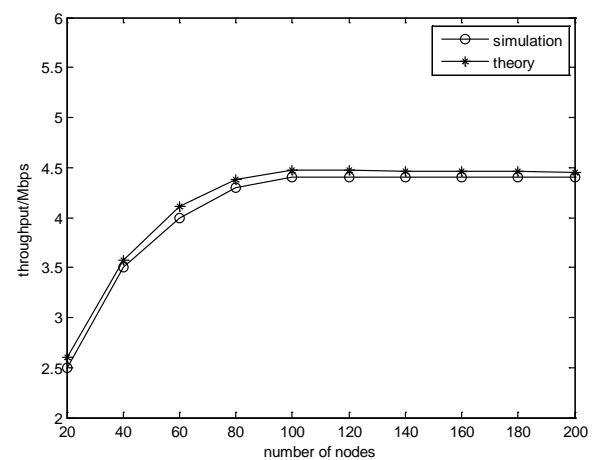

Fig. 6 Throughput results of simulation and theoretical analysis with estimation algorithm

Table 2 Computation delay with and without estimation algorithm

\begin{tabular}{ccccc}
\hline & 64Bytes & 128 Bytes & 256 Bytes & 1480 Bytes \\
\hline delay with algorithm & $1.2270 \mathrm{~s}$ & $1.2895 \mathrm{~s}$ & $1.3516 \mathrm{~s}$ & $1.5136 \mathrm{~s}$ \\
delay without algorithm & $1.2430 \mathrm{~s}$ & $1.4180 \mathrm{~s}$ & $1.4868 \mathrm{~s}$ & $1.6163 \mathrm{~s}$ \\
\hline
\end{tabular}

\section{Conclusion}

Although 2-dimension DTMC analysis model of DCF has been widely studied, both multi-hop analysis of MANETs and number evaluation algorithm of neighbor nodes haven't yet been investigated. In this paper, we first proposed a mutlihop model based on definition of ERR. Second, we qualitatively showed a key reason of deviation between theoretical analysis and simulation was number variation of neighbor nodes 
because of nodes mobility. Third, we realized a real-time estimation algorithm of neighbor nodes based on kalman filter via redefinition of status update and measurement rules in the assumption of poisson distribution. Last, we show that the extended model of DTMC achieves better performance. Since algorithm performance may be influenced by hidden terminal, which will be studied deeply in the future.

\section{Acknowledgment}

This work was jointly supported by the National Natural Science Foundation of China (No. 51075395, 61401499), the Industrial Science and Technology Project of Shaanxi Province (No. 2016GY-087).

\section{References}

[1] U Venkanna and R L Velusamy, "Black hole attack and their counter measure based on trust management in manet: a survey”, in Proc. of Advances in Recent Technologies in Communication and Computing, pp. 232-236, 2011.

[2] E Amiri, E Afshar and H R Naji, "Survey on network access control technology in MANETs", in Proc. of International Conference on Innovation Management and Technology Research (ICIMTR), pp.367-372, 2012.

[3] IEEE Standard for Wireless LAN Medium Access Control (MAC) and Physical Layer (PHY) Specifications, Nov., 1997, P802.11.

[4] D Eastlake 3rd, “IANA considerations and IETF protocol usage for IEEE 802 parameters: RFC5342” available online at http://www. rfc-editor.org/rfc/rfc5342.txt

[5] J Jang, S W Kim and S Wie, "Throughput and Delay Analysis of a Network Coding-enabled Cooperative MAC Protocol for Ad Hoc Networks," KSII Transactions on Internet and Information Systems, vol. 6, no. 6, pp. 1668-1688, 2012

[6] A Anjali, R Rinkle and Himanshu, "A Security Model based on Reputation and Collaboration through Route-Request in Mobile Ad Hoc Networks," KSII Transactions on Internet and Information Systems, vol. 9, no. 11, pp. 4701-4719, 2015.

[7] M Li, S Sergio, P Li, J Y Sun and X X Huang, "MAC-Lyer Selfish Misbehavior in IEEE 802.11 Ad Hoc Networks: Detection and Defense”, IEEE Transactions on Mobile Computing, vol.14, no.6, pp.1203-1217, 2015.

[8] Anjali Anand, Rinkle Rani and Himanshu Aggarwal, "Energy Efficient and Secure Multipoint Relay Selection in Mobile Ad hoc Networks," KSII Transactions on Internet and Information Systems, vol. 10, no. 4, pp. 1571-1589, 2016

[9] P J Gyanendra, Y N Seung, A Srijana and W K Sung, "Analysis of Channel Access Delay in CR-MAC Protocol for Ad Hoc Cognitive Radio Wireless Sensor Networks without a Common Control Channel," KSII Transactions on Internet and Information Systems, vol. 8, no. 3, pp. 911-923, 2014.

[10] M Haenggi, “The Local Delay in Poisson Network”, IEEE Transactions on Information Theory, vol. 59, no. 3, pp. 1788-1802, 2013.

[11] G Bianchi, "Performance analysis of the IEEE 802.11 distributed coordination function”, IEEE Journal on Selected Areas in Communications, vol. 18, no. 3, pp. 535-547, 2002

[12] Z H Gong, M Haenggi, “The Local Delay in Mobile Poisson Networks”, IEEE Transactions on Wireless Communications, vol. 12, no.9, pp. 4766-4776, 2013.

[13] R H Jia, F Yang, S C Yao, X H Tian, X B Wang, W J Zhang and J Xu, “Optimal Capacity Delay Tradeoff in MANETs with Correlation of Node Mobility”, IEEE Transactions on Vehicular Technology, vol. 66, no. 2, pp. 1772-1785, 2017

[14] J L Elvio and D Y Michel, "Exact Formulations for the Throughput of IEEE 802.11 DCF in Hoyt, Rice and Nakagami-m Fading Channels", IEEE Transactions on Wireless Communications, vol. 12, no.5, 2261-2271, 2013.

[15] T Navid, H G Wang and H H Chen, "Performance analysis of distributed access multihop poisson networks", IEEE Transactions on Vehicular Technology, vol. 63, no. 2, pp. 849-857, 2014

[16] B Uthman, A R Anas and M Samir, “The Impact of Sensor Node Distribution on Routing Protocols Performance: A Comparitive Study”, in Proc. of IEEE 11th International Conference on Trust, Security and Privacy in Computing and Communications, pp. 25-27, 2012.

[17] Y T Liu, J Xu and G Y Xia, “Node Distribution of the Random Direction Model in a Square Region”, in Proc. of International Conference on Mechatronics and Control (ICMC), pp.21-25, 2014

[18] S Nombuso, M Pragasen and O Olukayode, "Performance Evaluation of Routing Protocols in Uniform and Normal Node Distributions using Inter-Mesh Wireless Networks”, in Proc. of World Symposium on Computer Networks and Information Security (WSCNIS), pp1-6, 2015.

[19] C M Maricris, T Gregory and J L Nathaniel, “Development and Evaluation of Wave Sensor Nodes for Ocean Wave Monitoring”, IEEE Systems Journal, vol. 1, no. 9, pp. 292-302, 2015. 
[20] H H Chiang and Chiueh, “Accurate Clock Synchronization for IEEE 802.11 Based Multi-hop Wireless Networks”, in Proc. of IEEE International Conference on Networks Protocols, pp.11-20, 2009.

[21] A Muhammad and A H Syed, “A poisson point process model for coverage analysis of multi-hop cooperative networks”, in Proc. of International Wireless Communications and Mobile Computing Conference (IWCMC), pp. 442-447, 2015.

[22] C C Tseng and K C Chen, “On the distance distributions of the wireless ad hoc network”, in Proc. of IEEE Vehicle Technology Conference, vol. 2, pp.772-776, 2006.

[23] M Haenggi, “On distances in uniformly random networks”, IEEE Transactions on Information Theory, vol. 51, no. 10, pp. 3584-3586, 2005.

[24] M G Kendall and P A Moran, “Geometric Probability”, New York, NY, USA: Hafner, 1963.

[25] M Nakagami, “The m distribution-a general formula of intensity distribution of rapid fading”, in Proc. of Statistical methods in Radio Wave Propagtion, pp.3-36, 1960.

[26] A Baddeley, “Spatial Point Processes and Their Applications”, Berlin, Germany: Springer-Verlag, 2007.

[27] J Chung, “Claypool M. Ns By Example”, available online at http://nile.wpi.edu/NS/.

[28] G R Chen, “Kalman Filtering with Real-Time Applications(Fourth Edithion)”, Springer Series in Information Sciences, pp. 50-56, 2009. 